\title{
Non-Parametric Local Maxima and Minima Finder with Filtering Techniques for Bioprocess
}

\author{
K. K. L. B. Adikaram"1,2,3*, M. A. Hussein1, M. Effenberger², T. Becker ${ }^{4}$ \\ ${ }^{1}$ Group Bio-Process Analysis Technology, Technische Universität München, Freising, Germany \\ ${ }^{2}$ Bavarian State Research Center for Agriculture, Institute for Agricultural Engineering and Animal Husbandry, Freising, Germany \\ ${ }^{3}$ Computer Unit, Faculty of Agriculture, University of Ruhuna, Mapalana, Kamburupitiy, Sri Lanka \\ ${ }^{4}$ Lehrstuhl für Brau-und Getränketechnologie, Technische Universität München, Freising, Germany \\ Email: *lasantha@daad-alumni.de
}

How to cite this paper: Adikaram, K.K.L.B., Hussein, M.A., Effenberger, M. and Becker, T. (2016) Non-Parametric Local Maxima and Minima Finder with Filtering Techniques for Bioprocess. Journal of Signal and Information Processing, 7, 192-213. http://dx.doi.org/10.4236/jsip.2016.74018

Received: July 21, 2016

Accepted: October 8, 2016

Published: October 11, 2016

Copyright $\odot 2016$ by authors and Scientific Research Publishing Inc. This work is licensed under the Creative Commons Attribution International License (CC BY 4.0).

http://creativecommons.org/licenses/by/4.0/

\begin{abstract}
Typically extrema filtration techniques are based on non-parametric properties such as magnitude of prominences and the widths at half prominence, which cannot be used with data that possess a dynamic nature. In this work, an extrema identification that is totally independent of derivative-based approaches and independent of quantitative attributes is introduced. For three consecutive positive terms arranged in a line, the ratio $(\mathrm{R})$ of the sum of the maximum and minimum to the sum of the three terms is always $2 / n$, where $n$ is the number of terms and $2 / 3 \leq R \leq 1$ when $n=3$. R > $2 / 3$ implies that one term is away from the other two terms. Applying suitable modifications for the above stated hypothesis, the method was developed and the method is capable of identifying peaks and valleys in any signal. Furthermore, three techniques were developed for filtering non-dominating, sharp, gradual, low and high extrema. Especially, all the developed methods are non-parametric and suitable for analyzing processes that have dynamic nature such as biogas data. The methods were evaluated using automatically collected biogas data. Results showed that the extrema identification method was capable of identifying local extrema with $0 \%$ error. Furthermore, the non-parametric filtering techniques were able to distinguish dominating, flat, sharp, high, and low extrema in the biogas data with high robustness.
\end{abstract}

\section{Keywords}

Extrema Point, First Derivative, Peak Finder, Peaks and Valleys, Maxima and Minima, Second Derivative

\section{Introduction}

In process control, the method of determining peaks and valleys of a signal, also known as identification of local maxima and minima, is crucial for describing and capturing 
certain signal properties. Identification of local maxima and minima is particularly useful in signal processing, consequently useful in inline/online process control and optimization. Thereby, for reliable feature extraction it is necessary to remove redundant maxima and minima in a processed signal. The issue has been extensively investigated in literature [1]-[4], at which different techniques were reported. Magnitude-based methods and gradient-based methods are the most common two of such techniques. In magnitude based methods, the $n^{\text {th }}$ term of a series is $x_{n}$; $X_{n}$ is considered as a peak (maximum) when $x_{n-1}<x_{n}>x_{n+1}$. In the same time, $x_{n}$ is considered as a valley (minimum) when $x_{n-1}>X_{n}<x_{n+1}$. In gradient-based methods, extremum can be located by considering slope (gradient) of a certain point and acts as the most popular method [1]. When the slope is zero (first derivative is zero) at a certain point, the point can be described as a peak, valley or a saddle point. However, additional calculations are necessary to distinguish whether it is a peak, valley or saddle point. This encounter is solved by analysing the sign of the second derivative at the points of zero slopes [1]. The most popular methods of such are Newton Raphson method [2] and Taylor series-based derivatives [3] [4] which evaluate the derivatives numerically for a given data set.

Once the extrema points are identified, a filtration step is unavoidable to identify the dominant or relevant extrema. Magnitude of prominences and the widths at half prominence are two properties of signals that are commonly used to filter extrema [5] [6]. Furthermore, baseline correction is another technique used for finding out accurate maxima and minima [7]-[9]. In addition, there are numbers of methods for filtering unnecessary extrema based on template matching or masks [10], such as Kalman filters [11] [12] and non-linear filters [13]. Nevertheless, all aforementioned approaches are parametric methods [14], which question their robustness.

One of the main classifications existing in data analysis techniques is whether the method is parametric or non-parametric in its nature [15]. As mentioned above, most popular extrema filtering methods suffer from parametric concerns. Particularly, parametric methods use domain dependent value as detection criteria such as average, standard deviation, prominences of an extrema, and the widths at half prominence of an extrema. These criteria are based on domain dependent parameters and are therefore valid only for the considered data model or considered conditions in the domain. Thus, majorly parametric methods' accuracy inherits the variables' ranges and the conditions of the domain [16]. In reality, data capturing, especially within dynamic systems, such as biogas plants, is produced with various alterations. When the model or data range alters, whilst using parametric methods, it is necessary to recalibrate parameters or develop new models for monitoring, controlling, and data analysis, which is not of preference at process line.

\section{Non-Parametric Methods}

Non-parametric methods, also known as distribution-free methods, depend on fewer number of underlying assumptions [15] [17] [18], which progress them more as robust methods [16] [19]. In this research a new non-parametric technique for extrema identi- 
fication and filtration are developed. The proposed technique determines maxima and minima based on the relation of sum of terms in an arithmetic series. The same relation was used as a non-parametric method (MMS: a method based on maximum, minimum, and sum) for finding outliers in linear relation [20] and non-parametric linear fit identification method [21].

In some situations outliers, peaks and valleys are the same, when a sudden extremum (variation) occurs, additionally extrema can be formed due to gradual increment and gradual decrement. The extrema generated in such situations do not behave as outliers and cannot be identified using the aforementioned outlier detection method based on maximum, minimum, and data series sum (MMS) [20]. Furthermore, MMS can only be used for identifying outliers in liner regression and is not suitable for finding outliers in non-linear series [20]. This work focuses on modifying the methods of MMS for locating extrema in non-linear data series.

The proposed extrema identification method does not involve first or second derivative, but rather compares, within a considered window, two ratios in relation with maximum, minimum, middle point, and the sum of data points. Furthermore, three extrema filtration methods were introduced in this work, which are capable of filtering extrema independent of the prominences or width of an extremum. All the methods introduced in this work are developed for harsh conditions involved in dynamic processes, especially biogas process data, thus handling: non-linear datasets and based upon non-parametric methods.

\section{Materials and Methods}

As mentioned before, the outlier detection method, also by the same authors [20], will be modified to locate extrema in non-linear series. The method is based upon the theory of the sum of terms of an arithmetic progression. Having two major relations by means of $M M S_{\max }$ and $M M S_{\min }$ and are expressed in Equation (1) and Equation (2). The ratio $2 / n$ is used as the detection criteria, where $n$ is the number of terms in the series.

$$
\begin{gathered}
M M S_{\text {max }}=\left(a_{\text {max }}-a_{\min }\right) /\left(S_{n}-a_{\min } * n\right), \text { and } \\
M M S_{\text {min }}=\left(a_{\text {max }}-a_{\min }\right) /\left(a_{\max } * n-S_{n}\right),
\end{gathered}
$$

where $a_{\min }$ is the minimum element of the series, $a_{\max }$ is the maximum element of the series, $n$ is the number of terms in the series, and $S_{n}$ is the sum of terms in the series.

The complete expression for outlier detection is given by Equation (3). If any series expected to follow $y=c$ form and contains data that do not agree with $y=c$ form then:

$$
M M S=\left\{\begin{array}{l}
M S_{\text {max }}=\frac{a_{\max }-a_{\min }}{S_{n}-a_{\min } * n}=\left\{\begin{array}{l}
>(2 / n+w) ; \text { maximum is the outlier } \\
\leq(2 / n+w) ;-
\end{array}\right. \\
M S_{\text {min }}=\frac{a_{\max }-a_{\min }}{a_{\max } * n-S_{n}}=\left\{\begin{array}{l}
\leq(2 / n+w) ;- \\
>(2 / n+w) ; \text { minimum is the outlier }
\end{array}\right.
\end{array}\right.
$$

where $w$ is the weight.

The method $M M S$ expressed in Equation (3) can be applied on a window with any 
number of data points. However, when a window has only three data points it becomes a special situation, since the method generates an extremum when points are not in agreement with a linear fit, thus, if there is an extrema, always the middle point would be the extrema. When the numbers of data points are three $(n=3)$ and $w=0$, Equation (3) a special treatment is suggested:

$M M S=\left\{\begin{array}{l}M M S_{\text {max }}=\frac{a_{\text {max }}-a_{\text {min }}}{S_{3}-a_{\text {min }} * 3}=\left\{\begin{array}{l}>2 / 3 ; \text { Maximum is away from the other two points } \\ \leq 2 / 3 ;-\end{array}\right. \\ M M S_{\text {min }}=\frac{a_{\text {max }}-a_{\text {min }}}{a_{\text {max }} * 3-S_{3}}=\left\{\begin{array}{l}\leq 2 / 3 ;- \\ >2 / 3 ; \text { Minimum is away from the other two points }\end{array}\right.\end{array}\right.$

Equation (4) is a simplified version of Equation (3) for handling three data points, where $2 / 3 \leq M M S_{\max } \leq 1$ and $2 / 3 \leq M M S_{\min } \leq 1$. According to Equation (4), $M M S_{\max }>2 / 3$ implies that the maximum of the three points is always considerably apart from the other two points. In the same manner, $M M S_{\min }>2 / 3$ implies that the minimum of the three points is always considerably apart from the other two points. Plots (a), (b), and (c) of Figure 1 show situations that of $M M S_{\max }>2 / 3$, where maximum is the peak. Plots (e), (f), and (g) in Figure 1 show situations that of $M M S_{\min }>2 / 3$, where the minimum is the valley. However, Equation (4) does not always successfully identify extrema, in other words if the identified point is the first or last point of a window, theoretically it cannot be considered as an extrema. Plots (d)

\begin{tabular}{|c|c|c|c|c|c|c|}
\hline 120 \\
100 \\
80 \\
60
\end{tabular}

Figure 1. Plots (a), (b), (c), and (d) show different types of peaks and plots (e), (f), (g), and (h) show different types of valleys. For $n=3$, value $2 / n$ is 0.67 . In all peaks except plot (d) $M M S_{\max }>$ $2 / 3$ and in all valleys except plot (h) $M M S_{\min }<2 / 3$. " $\checkmark$ " corresponds to correct detections of extrema when MMS and $2 / n$ are used and " $\mathbf{~}$ " corresponds to wrong detections of extrema when MMS and $2 / n$ are used. Therefore, consideration of MMS and $2 / n$ is not a good method for identifying extrema. However, in the concept of outlier detection all the detections are correct. 
and (h) of Figure 1 show situations where neither a maximum nor a minimum represents an extremum. Figure 1(d) shows a peak that of $M M S_{\min }>2 / 3$ (identification of a valley), this is a contradicting situation. Also, Figure 1 (h) shows another failing situation, where the plot shows a valley that of $M M S_{\max }>2 / 3$ (identification of a peak). This occurs because in both considered situations, the point has the highest deviation is the first point and not the middle point. Therefore, Equation (4) is not capable of identifying extrema in such cases, thus handling these situations is required.

To address the aforementioned drawback, the MMS method was modified by considering the middle point of the window. To have an exact middle point in a data window the number of considered data points ( $n$ ) must be odd. When $n=3$ and $a_{\text {mid }}$ is the middle point of the window, substituting $a_{\max }$ from Equation (1) by $a_{\text {mid }}$ retrieves:

$$
M M S_{\text {max } \mid \text { mid }}=\left(a_{\text {mid }}-a_{\min }\right) /\left(S_{3}-a_{\min } * 3\right) .
$$

Also, by replacing $a_{\min }$ of Equation (2) by amid gives,

$$
M M S_{\text {min|mid }}=\left(a_{\max }-a_{\text {mid }}\right) /\left(a_{\max } * 3-S_{3}\right) .
$$

Consider the situation,

$$
\begin{aligned}
M M S_{\text {max }} & =M M S_{\text {max } \mid \text { mid }} . \\
\left(a_{\text {max }}-a_{\text {min }}\right) /\left(S_{3}-a_{\text {min }} * 3\right) & =\left(a_{\text {mid }}-a_{\text {min }}\right) /\left(S_{3}-a_{\text {min }} * 3\right), \\
a_{\text {max }} & =a_{\text {mid }} .
\end{aligned}
$$

Therefore, Equation (7) denotes the situation of a maximum at the middle point. Thus Equation (7) is a condition, independent of the value of MMS that can be used for identifying a peak.

Consider the situation:

$$
\begin{aligned}
M M S_{\text {min }} & =M M S_{\text {minlmid }} . \\
\left(a_{\text {max }}-a_{\text {min }}\right) /\left(a_{\text {max }} * 3-S_{3}\right) & =\left(a_{\text {max }}-a_{\text {mid }}\right) /\left(a_{\text {max }} * 3-S_{3}\right), \\
a_{\text {min }} & =a_{\text {mid }} .
\end{aligned}
$$

Then Equation (9) denotes the situation of a minimum at the middle point. Thus, Equation (9) is a condition, independent of the value of MMS that can be used for identifying a valley.

Therefore, when a window satisfies Equation (7) it implies that the middle point is a maximum and once a window satisfies Equation (9) it alternatively implies that the middle point is a minimum. Advancing the three point window by one data point makes it possible to locate all the extrema in a signal (Figure 2). Table 1 shows sample calculations of extrema detection procedure according to Equation (7) and Equation (9). The first eight value sets shown in Table 1 are the values in relation with the plots shown in Figure 1. Examples a, b, c, and d in Table 1 show calculation in relation with peak identification. In all these examples $M M S_{\max }=M M S_{\max \mid \operatorname{mid}}$ (Equation (7)) and $M M S_{\text {min }} \neq M M S_{\text {minlmid }}$ (Equation (9)). Examples e, f, g, and $\mathrm{h}$ in Table 1 show calculation in relation with valley identification. In all these examples $M M S_{\min }=M M S_{\text {min|mid }}$ (Equation (7)) and $M M S_{\max } \neq M M S_{\text {max mid }}$ (Equation (9)). The last two examples ( $i$ and 


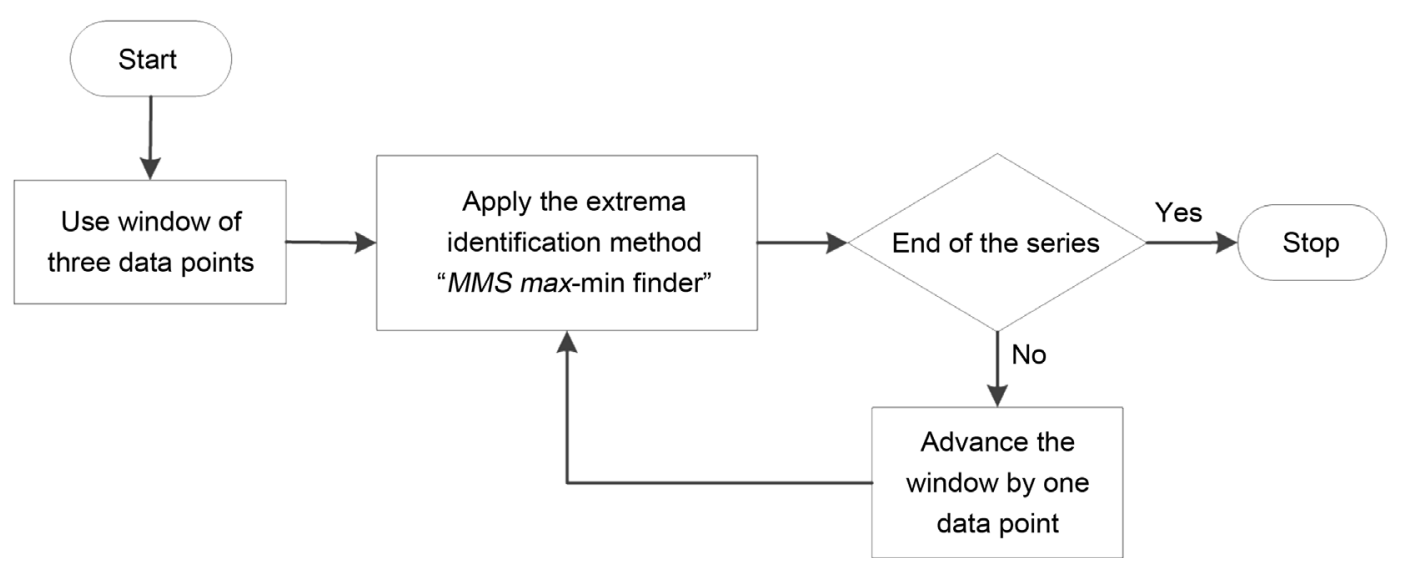

Figure 2. Extrema detection process of proposed extrema identification method named as MMS max-min finder.

Table 1. Sample calculations of peak and valley detection process based new method (MMS maxmin finder) for window size of three data points.

\begin{tabular}{ccccccccc}
\hline Plot & Data set & $\begin{array}{c}M M S_{\max } \\
(>0.67)\end{array}$ & $M M S_{\max / \operatorname{mid}}$ & $\begin{array}{c}M M S_{\min } \\
(>0.67)\end{array}$ & $M M S_{\min / \operatorname{mid}}$ & $\begin{array}{c}\text { Peak or } \\
\text { Valley }\end{array}$ & $\begin{array}{c}M M S_{\max }= \\
M M S_{\text {max/mid }}\end{array}$ & $\begin{array}{c}M M S_{\min }= \\
M M S_{\min / \operatorname{mid}}\end{array}$ \\
\hline (a) & $0,100,0$ & $1(\mathrm{Y})$ & 1 & $0.5(\mathrm{~N})$ & 0 & Peak & $\mathrm{Y}$ & $\mathrm{N}$ \\
(b) & $0,1.001,0$ & $1(\mathrm{Y})$ & 1 & $0.5(\mathrm{~N})$ & 0 & Peak & $\mathrm{Y}$ & $\mathrm{N}$ \\
(c) & $0,100,40$ & $0.714(\mathrm{Y})$ & 0.714 & $0.625(\mathrm{~N})$ & 0 & Peak & $\mathrm{Y}$ & $\mathrm{N}$ \\
(d) $\quad 0,100,90$ & $0.526(\mathrm{~N})$ & 0.526 & $0.909(\mathrm{Y})$ & 0 & Peak & $\mathrm{Y}$ & $\mathrm{N}$ \\
(e) $\quad 100,-20,100$ & $0.5(\mathrm{~N})$ & 0 & $1(\mathrm{Y})$ & 1 & Valley & $\mathrm{N}$ & $\mathrm{Y}$ \\
(f) & $-2,-2.2,-2$ & $0.5(\mathrm{~N})$ & 0 & $1(\mathrm{Y})$ & 1 & Valley & $\mathrm{N}$ & $\mathrm{Y}$ \\
(g) & $100,0,70$ & $0.588(\mathrm{~N})$ & 0 & $0.769(\mathrm{Y})$ & 0.769 & Valley & $\mathrm{N}$ & $\mathrm{Y}$ \\
(h) & $100,0,25$ & $0.8(\mathrm{Y})$ & 0 & $0.571(\mathrm{~N})$ & 0.571 & Valley & $\mathrm{N}$ & $\mathrm{Y}$ \\
(i) & $0,100,50$ & $0.667(\mathrm{~N})$ & 0.667 & $0.667(\mathrm{~N})$ & 0 & Peak & $\mathrm{Y}$ & $\mathrm{N}$ \\
(k) & $0,-100,-50$ & $0.667(\mathrm{~N})$ & 0 & $0.667(\mathrm{~N})$ & 0.667 & Valley & $\mathrm{N}$ & $\mathrm{Y}$ \\
\hline
\end{tabular}

k) show very special situations, where $M M S_{\max }, M M S_{\min }$, and $2 / n$ are equal. In such situations Equation (4) is undefined. However, even then extrema identification is possible with Equation (7) and Equation (9). Since the proposed extrema detection method is based on the maximum, minimum, and sum of the series, the method was named as “MMS max-min finder".

\subsection{Identifying Dominating Extrema (Primary Filtering of Peaks and Valleys)}

As above-mentioned, Equation (7) and Equation (9) are independent of the number of data points and thus valid for the situations where $\mathrm{n}$ is greater than three $(n>3)$. However to have an exact middle point, $n$ must be an odd number. When the numbers of data points are higher than three, there can be several peaks and several valleys. However, there is a situation that the highest peak (dominating peak) or lowest valley 
(dominating valley) coincides with the middle point of an advancing window. Figure 3 shows an example of detecting dominating peaks in a window with odd number of data points $(n=7)$. When the number of data points per window increases, it allows for the possibility of more than one extremum in the considered window.

The plot in Figure 3 consists of seven data points and contains three peaks named A, $\mathrm{B}$, and $\mathrm{C}$. The peak $\mathrm{A}$ is the middle point of window $W_{n}$ while peak $\mathrm{B}$ is the dominating peak. Because of that point $\mathrm{A}$ is not recognise as a peak in window $W_{n}$. After advancing $W_{n}$ by two data points, $W_{n+2}$ appears. In the window $W_{n+2}$ the point $\mathrm{B}$ is the highest as well as the middle point and the point $\mathrm{B}$ is recognized as a peak. Advancing $W_{n+2}$ by two data points $W_{n+4}$ appears, where $\mathrm{C}$ becomes the middle point and due to the influence of point $B$ it will not be recognized as a peak. This illustrates that the dominating extrema in a window remains undetected until the middle point of the window coincide with it whilst preventing identification of other small peaks and valleys.

The usage of windows with higher odd number of data points (e.g.: $5,7, \ldots)$ makes it possible to filter minor peaks and valleys. In contrast, if the methods in relation with height or width are used, the values are domain dependent and relative. Changing window size $(W)$ is an absolute parameter and can be applied in any condition, especially the situations that the domain conditions are unknown. However, this technique is not capable of filtering absolute small extrema, because the comparison is based on the existing extrema in the considered window. Furthermore, this technique is useful as a filter for removing relative small variations. Since the technique is based on the size of the window, the technique was named as "MMS-Window based filter" or (MMS-WBF).

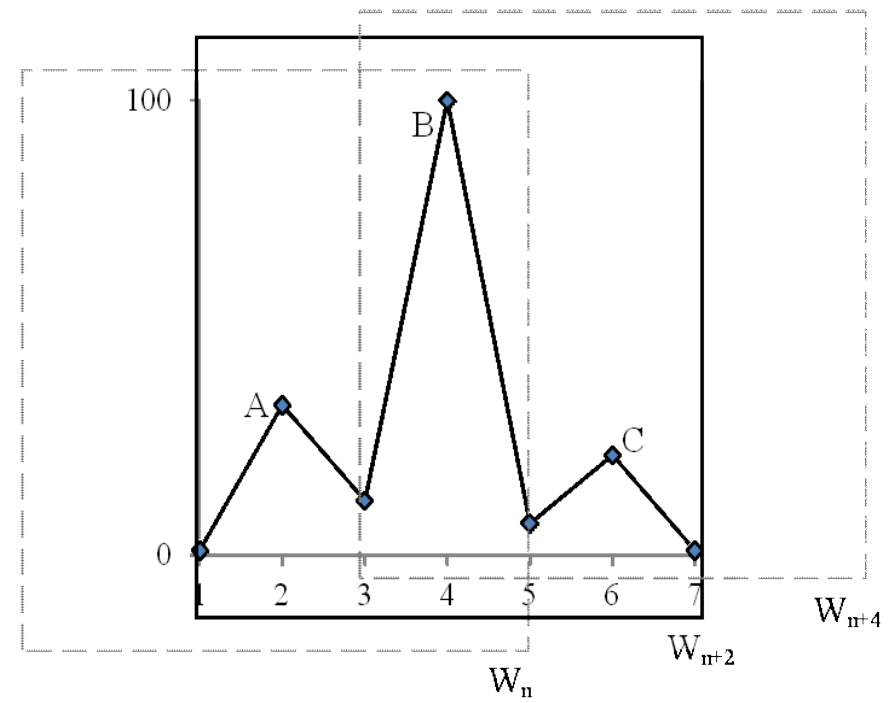

Figure 3. Application of "MMS max-min finder" with window size of seven for locating maximum point. In the window $W_{n}$ the middle point is " $\mathrm{A}$ " and due to existence of point " $\mathrm{B}$ " in the considered window, point " $\mathrm{A}$ " is not identified as the maximum point. Also, in the $W_{n+4}$ (the window found after advancing by four data points) the point " $\mathrm{C}$ " is not identified as an extrema, due to existence of point " $\mathrm{B}$ " in the considered the window. In the $W_{n+2}$ the point " $\mathrm{B}$ " is the maximum as well as middle point and there is no point larger than point " $\mathrm{B}$ " in the considered window. Thus, point " $\mathrm{B}$ " is identified as the dominating maximum. 


\subsection{Sharp and Gradual (Flat) Extrema Filtering}

Extrema with starting and end points which are agreeing with $y=c$ and having the middle point as the extremum can be considered as a symmetric extrema case. Plots (a) and (b) of Figure 4 show such symmetric extrema, which can be considered as the simplest symmetric form. Extrema shown in plots (c) and (d) of Figure 4 also fulfil the requirements of a perfect symmetric extrema. All the following equations in this section are based on the perfect extrema.

Consider a perfect maxima situation as shown in plot (c) of Figure 4. Here, all points are equal to $a_{\min }\left(a_{\min }=c\right)$ except $a_{\max }$. Consider any perfect maximum situation with $n$ points, then $n-1$ points are equal to $a_{\min }$, and $a_{\max } \neq c$. The sum of the terms of such a series can be expressed as:

$$
\begin{aligned}
& S_{n}=a_{\min } *(n-1)+a_{\max } \\
& \left(S_{n}-a_{\max }\right) / a_{\min }=(n-1)
\end{aligned}
$$

Consider a perfect minimum situation as shown in plot (d) of Figure 4. Here, all points of the series are equal to $a_{\max }$ and $a_{\max }=c$ except $a_{\min }$. Consider any perfect maxima situation with $n$ points. Then $n-1$ points are equal to $a_{\max }$, and $a_{\min } \neq c$. The sum of the terms of such a series can be expressed as:

$$
\begin{aligned}
& S_{n}=a_{\max } *(n-1)+a_{\min } \\
& \left(S_{n}-a_{\min }\right) / a_{\max }=(n-1)
\end{aligned}
$$

If $M M S_{\max } / M M S_{\min }=R_{M m}$, then from (1) and (2),

$$
R_{M m}=\left(a_{\max } * n-S_{n}\right) /\left(S_{n}-a_{\min } * n\right) \text {. }
$$

When the maximum is detected as the peak, substituting in Equation (11) retrieves:

$$
\begin{gathered}
R_{M m}=\left(a_{\max } * n-\left(a_{\min } *(n-1)+a_{\max }\right)\right) /\left(\left(a_{\min } *(n-1)+a_{\max }\right)-a_{\text {min }} * n\right) \\
R_{M m}=\left(a_{\max } * n-\left(a_{\min } * n-a_{\min }+a_{\max }\right)\right) /\left(\left(a_{\min } * n-a_{\min }+a_{\max }\right)-a_{\text {min }} * n\right) \\
R_{M m}=\left(\left(a_{\max }-a_{\min }\right) * n-\left(a_{\max }-a_{\min }\right)\right) /\left(a_{\max }-a_{\min }\right)
\end{gathered}
$$

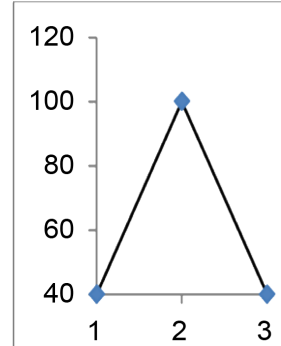

(a)

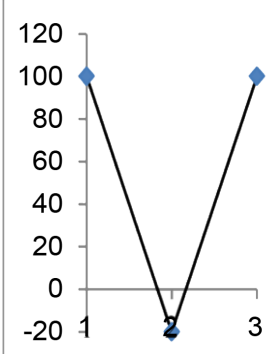

(b)

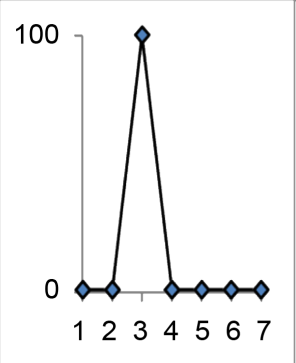

(c)

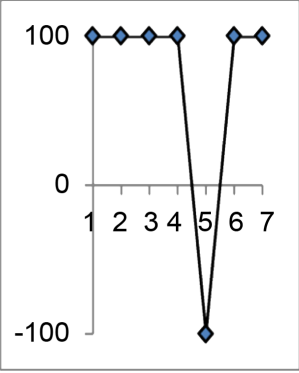

(d)

Figure 4. Perfect extrema. A perfect extrema is defined as an extrema that is symmetric extrema. Thus, in a perfect extrema both the starting and end points follow the $y=c$ form. Plots (a) and (b) show the simplest perfect extrema and plots (c) and (d) show perfect extrema that have more points that agree with $y=c$ form. 


$$
\begin{gathered}
R_{M m}=\left(\left(a_{\text {max }}-a_{\text {min }}\right) *(n-1)\right) /\left(a_{\text {max }}-a_{\text {min }}\right) \\
R_{M m}=(n-1) \\
M M S_{\text {max }} / M M S_{\text {min }}=(n-1)
\end{gathered}
$$

In the same manner, if $M M S_{\min } / M M S_{\max }=R_{m M}$, then from Equations (1), (2), and (12), the minimum is detected as the valley,

$$
\begin{gathered}
R_{m M}=(n-1) \\
M M S_{\min } / M M S_{\max }=(n-1)
\end{gathered}
$$

The relations of Equation (15) and Equation (16) are crucial findings, which can be used to identify perfect extrema. When the extrema is not perfect, value of Equation (15) and Equation (16) is less than $n-1$. Therefore, Equation (15) and Equation (16) can be used to identify perfect and non-perfect extrema. Also, perfect extrema are sudden (sharp) extrema and non-perfect extrema can be considered as gradual extrema. Thereby, using Equation (15) and Equation (16) it is possible to filter sharp and gradual extrema.

After identifying a peak, by examining the ratio $M M S_{\max } / M M S_{\min }$ it is possible to determine degree of confidence of other points, the same applies for identifying a valley. Assume $t_{M m_{-} m M}$ is the threshold value for determining sharp and gradual maxima, then $t_{M m_{-} m M}$ can be expressed as a $k *(n-1)$, where $0<k \leq 1$. If $k$ is expressed as a function of $n$ (e.g.: $k=1 /(n-1))$, then $t_{M m_{-} m M}$ is a function of $n$. By setting the same threshold value $\left(t_{M m_{\_} m M}\right)$ for $M M S_{\max } / M M S_{\min }$ and $M M S_{\min } / M M S_{\max }$, sudden and gradual maxima can be determined. The determination criteria $\left(t_{M m_{-} m M}\right)$ of ratios $M M S_{\max } / M M S_{\min }$ and $M M S_{\min } / M M S_{\max }$ are non-parametric and depend only on the number of data points in the considered window. Since the method is also based on the maximum, the minimum, and the sum, the method was named as MMS-SG filter.

Figure 5 and Figure 6 show examples in relation with Equation (15) and Equation (16), respectively. In plots (a) and (b) of Figure 5, the ratio $M M S_{\max } / M M S_{\min }=6$, which is exactly equal to $n-1$. This proves the correctness of Equation (15). In the same time, in plots (a) and (b) of Figure 6, the ratio $M M S_{\min } / M M S_{\max }=6$ and proves the correctness of Equation (16). All these plots exhibit either sudden peak or sudden valley. The corresponding ratios in relation with the plot (c) of Figure 5 and Figure 6 are not equal to $n-1$. However, the corresponding ratios are not very small. Therefore, these extrema can be considered as nearly sharp extrema. Nevertheless, corresponding ratios in relation with, plots (d) of Figure 5 and Figure 6 are very small and these extrema can be considered as gradual extrema.

\subsection{High and Low Extrema Filtering}

MMS-WBF and MMS-SG introduced in this work are capable identifying dominating, sharp and gradual extrema. However, these techniques are incapable of distinguishing the extrema with very small amplitude as shown in Figure 1(b) and Figure 1(f).

The valley shown in Figure 7 is a general situation of a perfect valley. When a valley has a very small crater, $a_{\min } \approx a_{\max }$. 


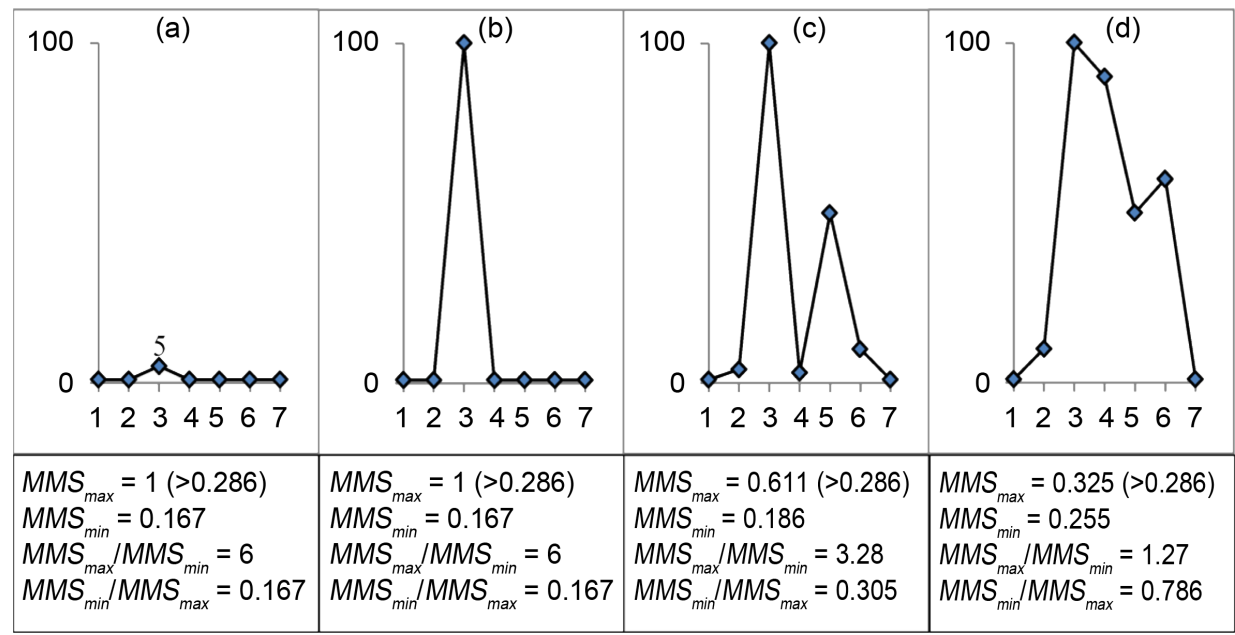

Figure 5. Plots (a), (b), (c), and (d) show four different types of peaks with window size seven $(n=$ 7) where $2 / n=0.286$. Ratios $M M S_{\max } / M M S_{\min }$ and $M M S_{\min } / M M S_{\max }$ are stated along with each plot. Peaks in plots (a) and (b) are perfect peaks and the ratio $M M S_{\max } / M M S_{\min }=6$ (i.e. $\left.n-1\right)$. Though, the dominating peak in plot (c) is not a perfect peak, ratio $M M S_{\max } / M M S_{\min }$ is consideribly high. The peak in plot (d) is a gradually developed peak and also not a perfect peak and the ratio $M M S_{\max } / M M S_{\min }$ is very small. Therefore, consideration of ratio $M M S_{\max } / M M S_{\min }$ is a good criterion to distinguish sudden and gradual peaks.

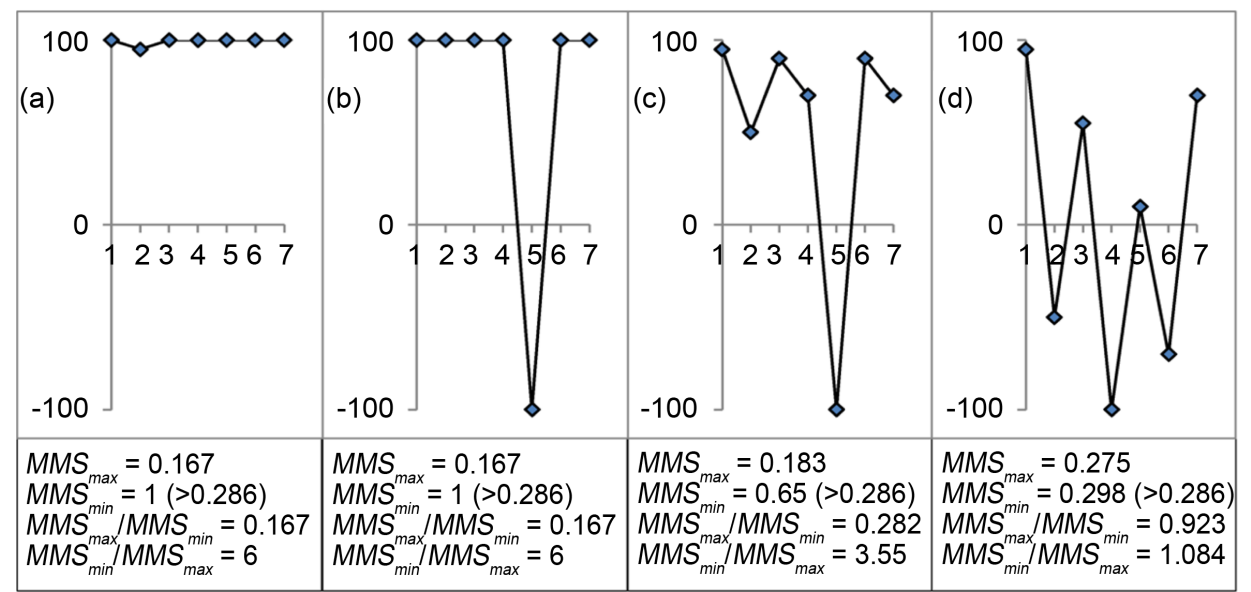

Figure 6. Plots (a), (b), (c), and (d) show four different types of valleys with window size seven $(n=$ 7) where $2 / n=0.286$. Ratios $M M S_{\max } / M M S_{\min }$ and $M M S_{\min } / M M S_{\max }$ are stated along with each plot. Valleys in plots (a) and (b) are perfect valleys and the ratio $M M S_{\min } / M M S_{\max }=6$ (i.e. $\left.n-1\right)$. Though, the valley in plot (c) is not a perfect valley, ratio $M M S_{\min } / M M S_{\max }$ is considerably high. The valley in plot (d) is a gradually developed valley and also not a perfect valley and the ratio $M M S_{\min } / M M S_{\max }$ is very small. Therefore, consideration of ratio $M M S_{\min } / M M S_{\max }$ is a good criterion for distinguishing between sudden and gradual (flat) valleys.

Then, Equation (13) can be expressed as:

$$
\begin{gathered}
S_{n} \approx a_{\min } *(n-1)+a_{\min } \\
S_{n} \approx a_{\min } * n ;\left(<a_{\max } * n\right) \\
R_{L_{-} \text {min }}=\left(a_{\min } * n\right) / S_{n} ; 0<R_{L H_{-} \min } \leq 1
\end{gathered}
$$




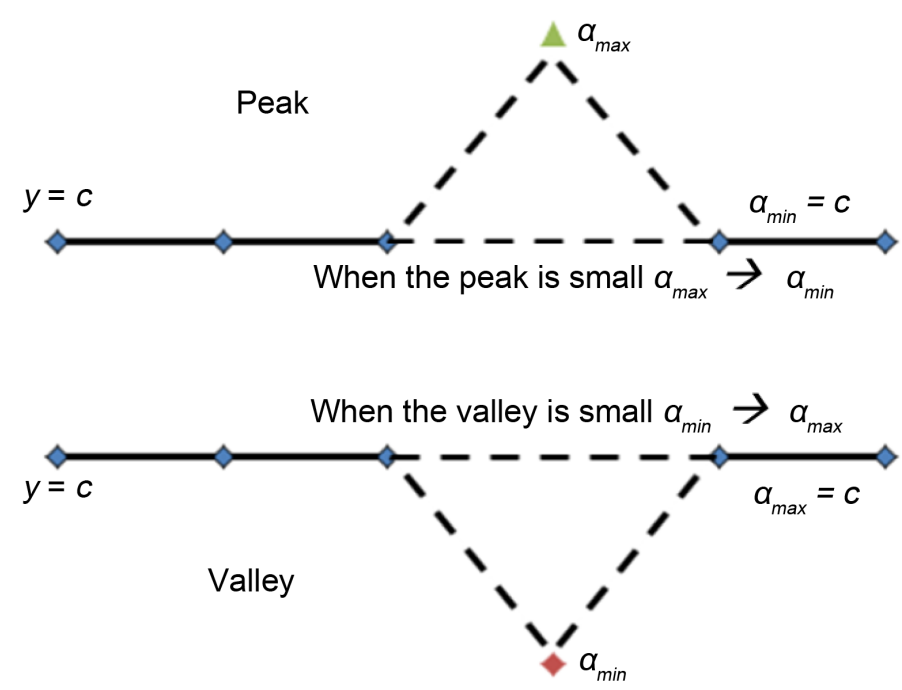

Figure 7. Two possible ways of existence of extremum for a data point; as a peak or as valley. Assume, except the extremum, all the other points are satisfying the $y=c$ relation (perfect extrema). Then, extremum is the peak and all other points are equal to the minimum. In the same manner, when the extremum is a valley, valley is the minimum and all other points are equal to the maximum. If a peak is small it reaches to the minimum and when the valley is small it reaches to the maximum. This is the hypothesis for distinguishing small and high extrema.

If $R_{L H_{-} \min } \rightarrow 1$, it implies that the $a_{\min }$ is very close to the other points (low crater), consequently $R_{L H_{-} \min } \rightarrow 0$ implies that the $a_{\min }$ is apart from the other points (high crater).

In Equation (17), when the term $a_{\min }$ is zero, the ratio $R_{L H \_ \text {min }}$ also becomes zero despite of the influence of magnitude valley. Also, due to the influence of negative values $S_{n}$ can be zero and $R_{L H_{-} \min }$ becomes invalid. Both these situations inhibit the determination of the real condition of the valley. To overcome the effect of negative values, the minimum value was deducted from all the terms of the data points in the window as expressed in Equation (18).

$$
a_{i_{-} \text {New }}=a_{i}-a_{\text {min }} .
$$

Even now it is possible to have a situation of $a_{\min }=0$. To overcome this situation a constant $k$, which is greater than zero, was added to each value. This transformation is applied in "Min-Max normalization" process [22] [23]. When $k=1$ thus Equation (18) becomes:

$$
a_{i_{-} \text {New }}=a_{i}-a_{\min }+1
$$

From Equation (17) and Equation (19),

$$
\begin{gathered}
R_{L H_{-} \min }=\left(\left(a_{\min }-a_{\min }+1\right) * n\right) / \sum_{i=1}^{n}\left(a_{i}-a_{\min }+1\right) \\
R_{L H_{-} \min }=n /\left(\sum_{i=1}^{n} a_{i}-\sum_{i=1}^{n} a_{\min }+\sum_{i=1}^{n} 1\right) \\
R_{L H_{-} \min }=n /\left(S_{n}-a_{\min } * n+n\right) ; 0<R_{L H_{-} \min } \leq 1
\end{gathered}
$$




$$
R_{L H_{-} \min }=n /\left(S_{n}+\left(1-a_{\min }\right) * n\right) ; 0<R_{L H_{-} \min } \leq 1
$$

Then $R_{L H_{-} \min }$ expressed in Equation (20) can be considered as a robust method for filtering valleys with low crater.

The peak shown in Figure 7 is a general situation of perfect peak. When a peak has a very small prominence, $a_{\max } \approx a_{\min }$. Then Equation (11) can be expressed as:

$$
\begin{gathered}
S_{n} \approx a_{\max } *(n-1)+a_{\max } \\
S_{n} \approx a_{\max } * n ;\left(>a_{\min } * n\right) \\
R_{L H_{-} \max }=\left(a_{\max } * n\right) / S_{n} ;>0
\end{gathered}
$$

According to Equation (17), the ratio $R_{L H_{-} \min }$ has a well-defined upper limit (ceiling) and lower limit (floor) because $0<R_{L H_{-} \min } \leq 1$. Nevertheless, in Equation (21), $R_{L H_{-} \max }$ has no upper limit, and subjects only to a lower limit. Therefore, it is difficult to use $R_{L H_{-} \max }$ as a global criteria as $R_{L H_{\_} \text {min. }}$. The peak shown in Figure 7 can be considered as the mirror image of a valley in Figure 7. Thus, it is possible to transform a peak to a valley, for that Equation (17) can be used for determining the peaks with high and low prominence using the same criteria Under the assumption that:

$$
a_{i_{-} \text {New }}=\left(a_{\max }+a_{\text {min }}\right)-a_{i}
$$

According to Equation (22), $\left(a_{\max }+a_{\min }\right)-a_{\max }=a_{\min }$ and $\left(a_{\max }+a_{\min }\right)-a_{\min }=a_{\max }$. The expression in Equation (22) transforms the maximum value into the minimum, the minimum value into the maximum and intermediate values into their complements. If the $R_{L H_{-} \max }$ is the corresponding ratio in relation with high and low peaks identification, then, from Equation (21) and Equation (22), one can reach:

$$
\begin{gathered}
R_{L H_{-} \max }=\left(\left(\left(a_{\max }+a_{\min }\right)-a_{\max }\right) * n\right) / \sum_{i=1}^{n}\left(\left(a_{\max }+a_{\min }\right)-a_{i}\right) \\
R_{L H_{-} \max }=\left(a_{\text {min }} * n\right) / \sum_{i=1}^{n}\left(\left(a_{\text {max }}+a_{\text {min }}\right)-a_{i}\right)
\end{gathered}
$$

Even after the aforementioned transformation, it is still possible to have the influence of negative values. However, it can be resolved by using Equation (19). Then, from Equation (19),

$$
\begin{gathered}
R_{L H_{-} \max }=\left(\left(a_{\min }-a_{\min }+1\right) * n\right) / \sum_{i=1}^{n}\left(\left(a_{\max }-a_{\min }+1+a_{\min }-a_{\min }+1\right)-\left(a_{i}-a_{\min }+1\right)\right) \\
R_{L H_{-} \max }=n / \sum_{i=1}^{n}\left(\left(a_{\max }+1\right)-a_{i}\right) \\
R_{L H_{-} \max }=n /\left(\left(a_{\max }+1\right) * n-S_{n}\right) ; 0<R_{L H_{-} \max } \leq 1
\end{gathered}
$$

$R_{L H_{-} \max } \rightarrow 1$ implies that the $a_{\max }$ is very close to other points (low prominence). Consequently $R_{L H_{-} \max } \rightarrow 0$ implies that the $a_{\max }$ is apart from the other points (high prominence).

Finally, using Equation (17) and Equation (24) it is possible to determine the high and low extrema by defining a threshold value $t_{L H}\left(0<t_{L H} \leq 1\right)$ for $R_{L H_{-} \min }$ and $R_{L H_{-} \max }$. 
Because the method is based on the maximum, minimum and the sum, the method was named as MMS-LH. Figure 8 elaborates the functionality of MMS-LH as a filtering method.

The filtration of sudden, gradual, low, and high extrema are derived based on a data set which satisfies the $y=c$ relation (perfect extrema). However, in reality it is impossible to always have perfect extrema. Therefore, by setting the threshold values in appropriate situations, it is possible to filter the extrema in non-perfect conditions.

Extrema identification is performed after comparing two ratios in relation with maximum, minimum, middle point and sum. The threshold criteria for MMS-WBF and MMS-SG are values that are based on the number of data points $(n)$. The threshold criterion for MMS-LH is a value between 0 and 1. Thus, all the determination criteria are totally non-parametric. However, combination of these methods leads to harvest more robust and reliable output. Figure 9 elaborates one possibility of combining all these methods for achieving reliable output.

All the algorithms were implemented using $\mathrm{C}++$ in Net 2008 platform and tested with biogas data which were collected online form a biogas plant using NIR spectroscopy for a period of seven months with a frequency of twelve data points per day (i.e. every second hour). Among the different parameters, the $\mathrm{H}_{2}$ content measured in ppm was selected, which has considerable amount of variations during the process. Data of each month was considered as a segment, where each segment consists of $350-400$ data points. The proposed detection methods were applied on each segment with different criteria. Furthermore, another data set of around 4800 data points, concentrations of volatile fatty acid (VFA), was selected for checking segmenting capabilities of the method.

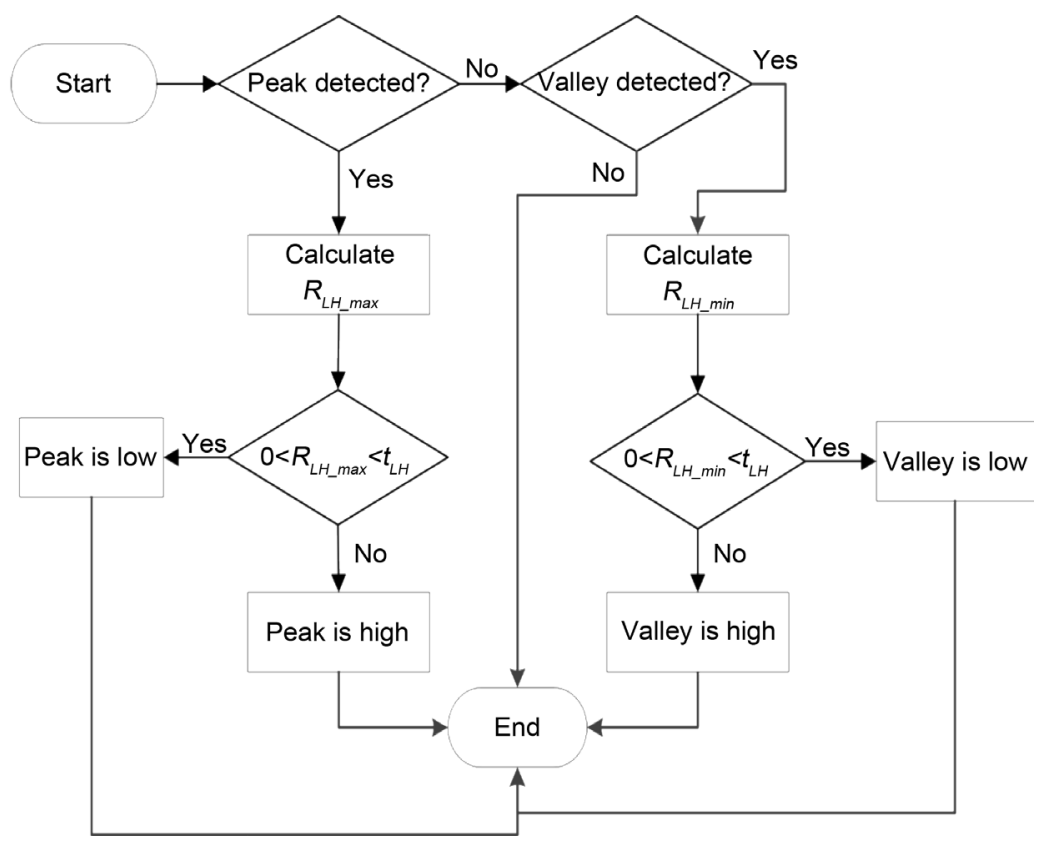

Figure 8. High and low extrema detection algorithm for "MMS-LH filter". Compression of predefined threshold $t$ for $R_{L H_{-} \max }$ and $R_{L H_{-} \min }$ allows distinguishing low and high extrema. 


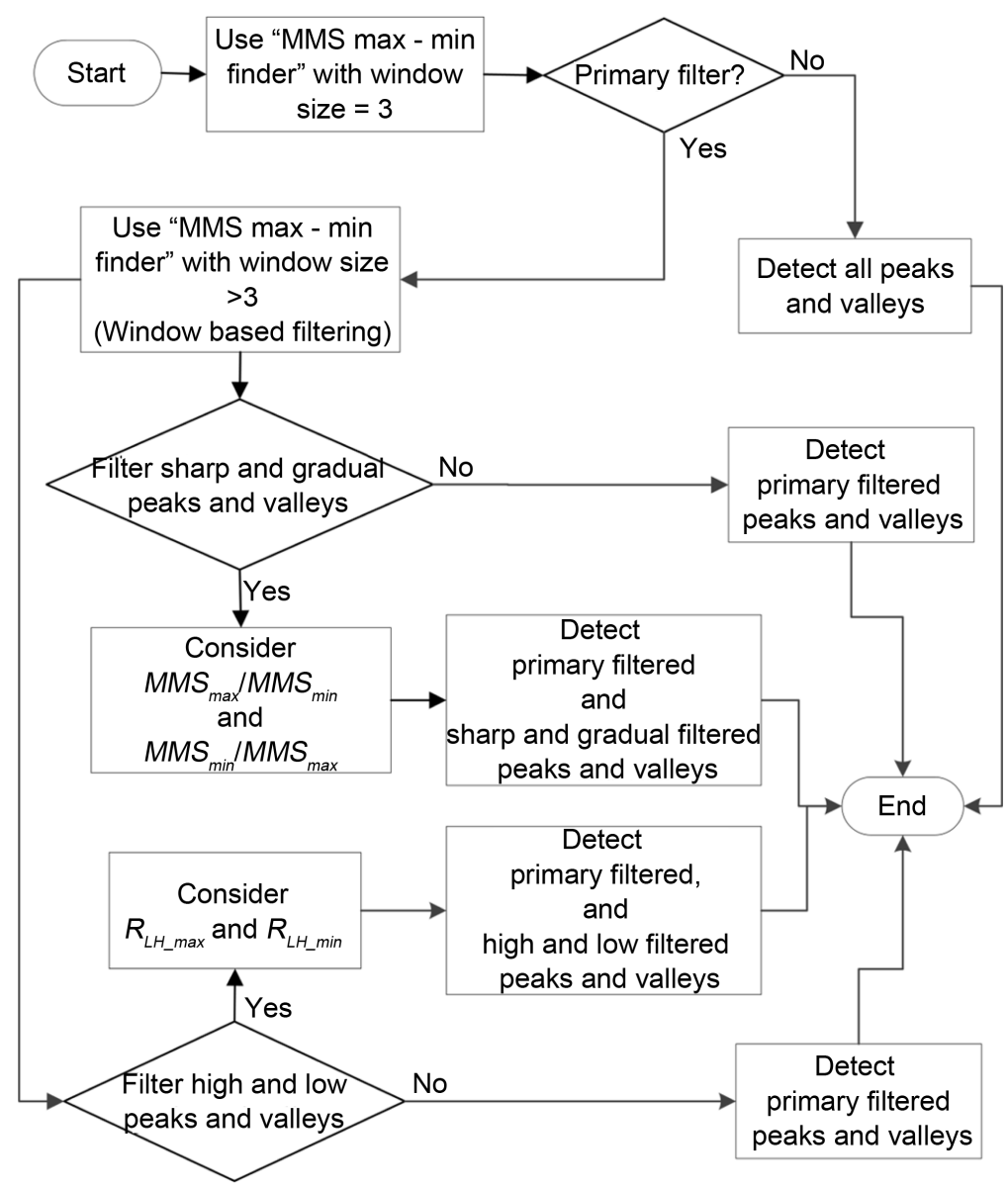

Figure 9. One possible way of combining all the developed methods for harvesting quality output.

\section{Results and Discussion}

\subsection{Identifying Extrema}

Each plot (a) and (b) of Figure 10 contains between 350 and 400 data points and shows the identified extrema using the proposed "MMS max-min finder", which is based on Equation (7) and Equation (9). In both situations all the extrema were detected with a window size of three $(W=3)$, which is the smallest valid size of the window. Results show detection of all the extrema with $0 \%$ error. However, there is an interesting feature about detections, which can be sometimes defined as an incorrect detection as seen in Figures 10(c)-(f). Plot (c) and (d) of Figure 10 show the case where two consecutive maxima with the same value and two consecutive minima with the same value, respectively. When $W=3$, usually both the adjacent extrema of a certain extremum have opposite extremum type (e.g.: for a maximum, adjacent members are two minima). If one adjacent extremum is with the same type extremum (e.g.: for a maximum, one adjacent member is a maximum) implies that the intermediate points of relevant points have the same value ((d) of Figure 10). Using the same criteria these detections can be excluded, if necessary. 


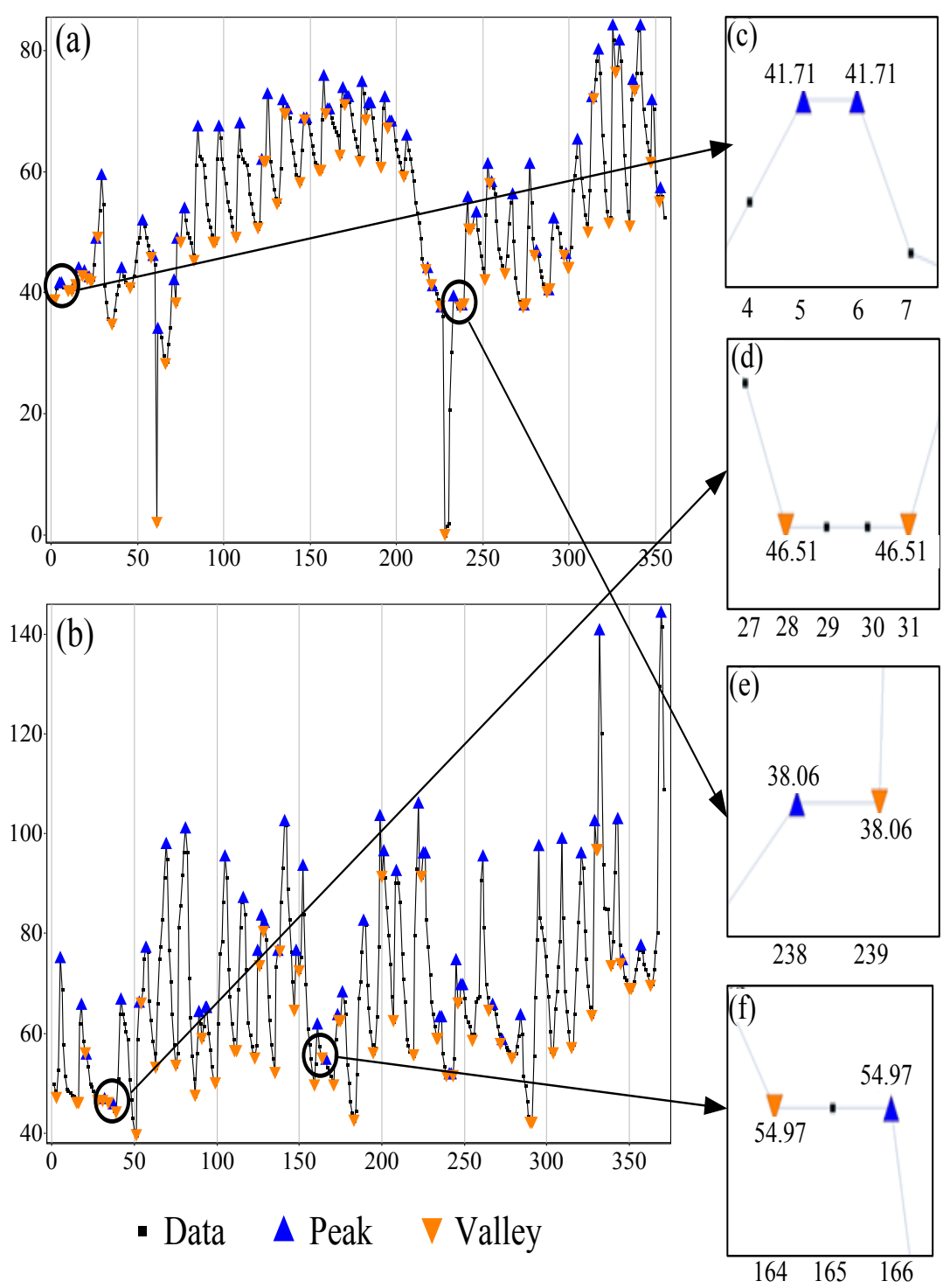

Figure 10. Two plots of $\mathrm{H}_{2}$ content of biogas data in two different months are presented measured in ppm. All the maxima in both the data sets ((a) and (b)) were identified by the new method with the window side is three $(W=3)$. Plots (c), (d), (e), and (f) show identification of special situations as extrema, even though they are existing derivative methods not consider as extrema situations.

Plot (e) and (f) of Figure 10 show other different situations, where it has consecutive minima and maxima of the same value. This also implies that the intermediate points have the same value ( $(\mathrm{f})$ of Figure 10). If consecutive maxima have same values and the order of occurrence is maximum then minimum, it can be considered as a discrete saddle region in an increasing data segment ((e) of Figure 10). In the same manner, if the two consecutive extrema have same value and the order of occurrence is minimum then maximum, it can be considered as a discrete saddle region in a decreasing data segment ((f) of Figure 10). Using the same criteria these detections can be excluded, if necessary. 


\subsection{Identifying Dominating Extrema (Primary Filtering of Peaks and Valleys)}

The same two data sets shown in Figure 10 were filtered using MMS-WBF (MMS Window based filtering) method for identifying the dominant extrema using a window size of $9(W=9)$. Results of the detection process are shown in Figure 11 plots (a) and (b) demonstrate that the MMS-WBF was capable to identify $50 \%$ and $59 \%$ of all extrema as dominating extrema, respectively. However, out of the identified extrema in plots (a) and (b), there are $0.12 \%$ and $0.09 \%$ of small peaks which are identified as dominating extrema. These extrema cannot be visually justified as dominating extrema. Nevertheless, numerically they are the dominating extrema in the considered window size. One possible option is to increase the window size, thus covering more data which enhances the capability of removing more non-dominating extrema. However, when $W>3$, all
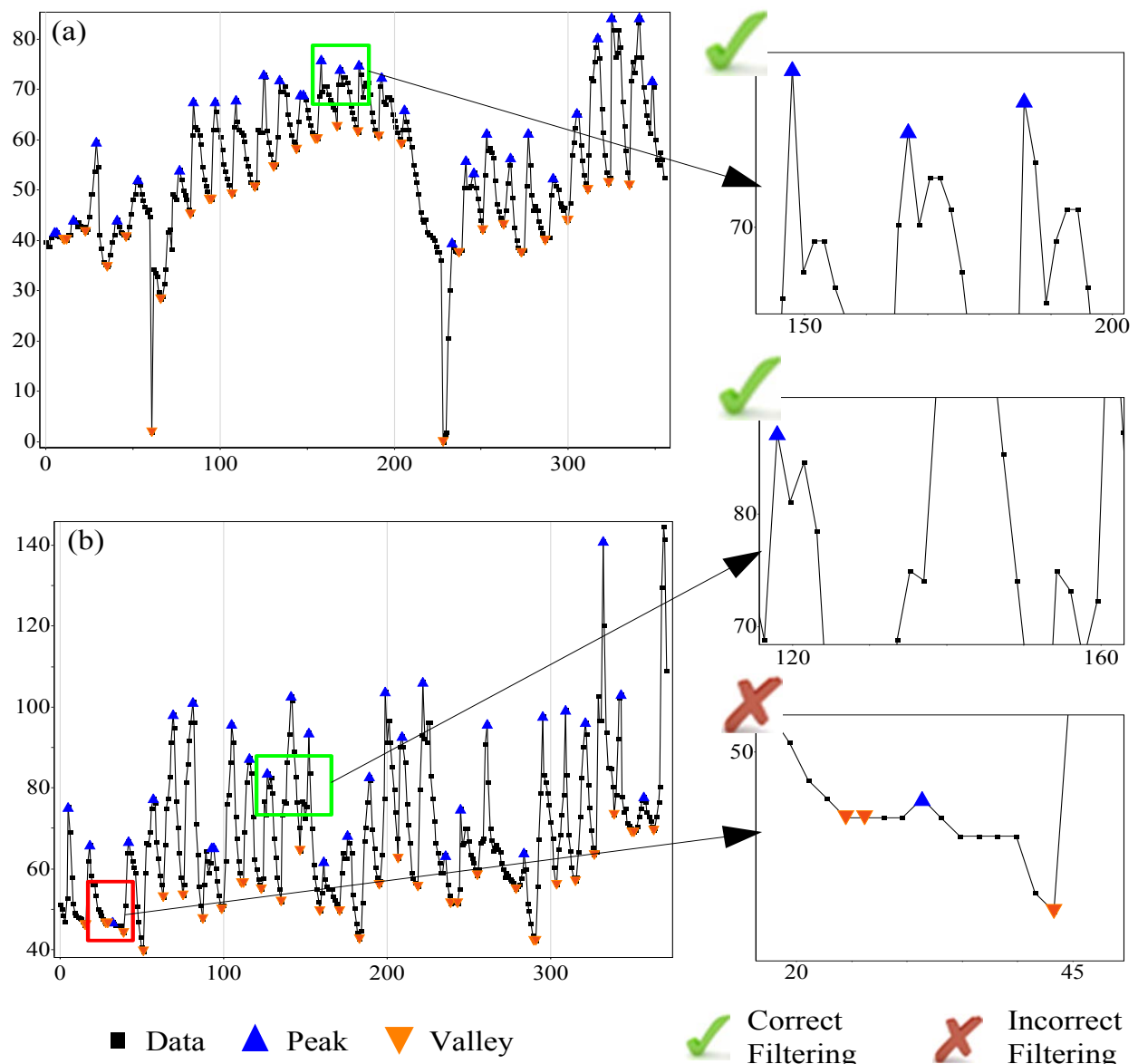
the candidate points have not been checked. This is a disadvantage of increasing the window size for filtering non-dominating extrema. In plot (d) of Figure 11, at the end of the data set shows such an unidentified dominating peak due to $W>3$ situation.

The combination of MMS max-min finder and MMS-WBF can be used in online data checking. For that, first the window size $(W)$ has to be defined, and then the window accumulates the data, after which the desired detection technique is applied and eventually the extrema are located. Subsequently, window is advanced by one data point and awaits the next data point. After the next point is captured, the extremacheck is performed again. This process is propagated throughout the process for locating extrema in an online environment.

\subsection{Sharp and Gradual (Flat) Extrema Filtering}

Figure 12 shows the results in relation with sharp and gradual extrema detection performed based upon $R_{M m}$ and $R_{m M}$ as defined in Equation (15) and Equation (16), respectively. Value of $t_{M m_{-} m M}$ for $R_{M m}$ and $R_{m M}$ was set as $1(k=1 /(n-1))$. Plot (a) and (b) of Figure 12 show the filtering of extrema, first with MMS-WBF for $W=3$ and then with MMS-SG filter. Plot (c) and (d) of Figure 12 shows the filtering of extrema with MMS-WBF in the case of a window size of $9(W=9)$ and then with MMS-SG filter. When compared, plots (a) and (b) of Figure 12 show $78 \%$ and $77 \%$ less number of all extrema than number of extrema shown in plots (a) and (b) of Figure 10. When the $W$ is small ( $W=3$ ) filter excludes some extrema seems to be very high $\left(\mathrm{V}_{1}, \mathrm{P}_{1}, \mathrm{P}_{2}\right.$, and $\mathrm{P}_{3}$ shown in plots (a) and (b) of Figure 12), which can be considered as wrong detection. However, according to Equation (11) and Equation (13), rejections of those points are mathematically correct. This happens due to usage of small window size for extrema detection. Thus, one solution for overcoming this situation is to use lager window size.

Plots (c) and (d) in Figure 12 show identification of $\mathrm{V}_{1}, \mathrm{P}_{1}, \mathrm{P}_{2}$, and $\mathrm{P}_{3}$ after increasing the window size to nine ( $W=9)$. After applying large $W(W=9)$ almost all the flat extrema have been rejected. Even after increasing the $W$ still extrema such as $\mathrm{P}_{4}$ are remaining, because $W$ is not big enough to reject such points (i.e. in the selected window size, the extremum point is located significantly away from other points). In general, plots (c) and (d) of Figure 12 show $0.46 \%$ and $0.75 \%$ fewer extrema in comparison with plots (a) and (b) of Figure 12 and all the detections and rejections are agreed with the developed method. Therefore, the ratios $M M S_{\max } / M M S_{\min }$ and $M M S_{\min } / M M S_{\max }$ can be considered as filtering criteria and a reliable technique for filtering sharp and gradual (flat) extrema.

\subsection{High and Low Extrema Filtering}

As per the results shown in Figure 10 and Figure 12 it is very clear that the "primary filtering" and consideration of $M M S_{\max } / M M S_{\min }$ and $M M S_{\min } / M M S_{\max }$ are not capable of filtering extrema based on magnitude of their prominence or crater. The results shown in Figure 13 are the results in relation with the method MMS-LH, which is intensively developed focusing on filtering extrema with low prominence or crater. 

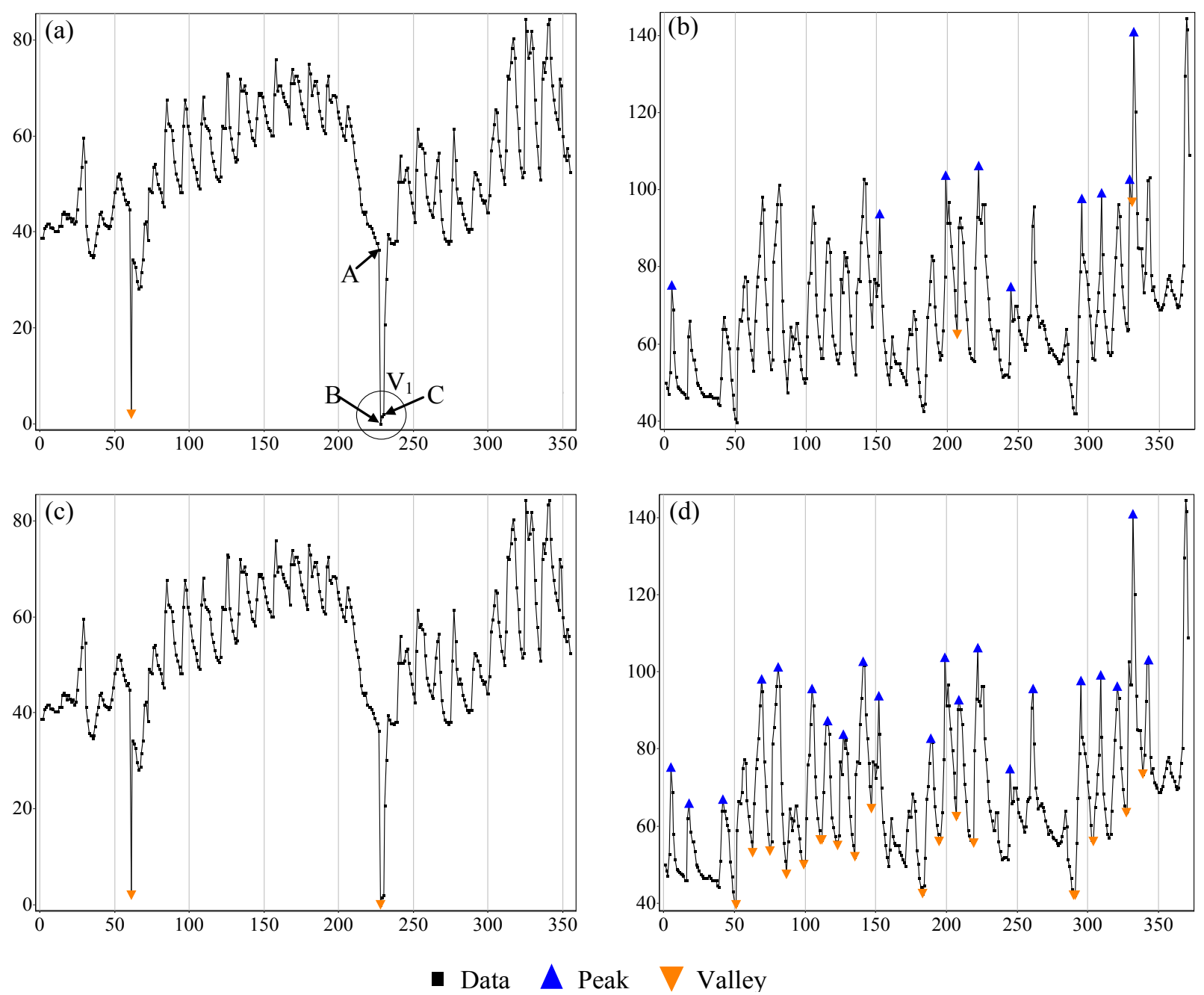

Figure 12. Filtering of sudden and gradually developed (flat) extrema using MMS-SG technique. Data in plots (a) and (b) were first checked for extrema with a window of size three with MMS-WBF. Data in plots (c) and (d) were first checked for extrema with a window of size nine with MMS-WBF. Then ratios $M M S_{\max } / M M S_{\min }$ and $M M S_{\min } / M M S_{\max }$ considered and all the plots were checked for sudden and gradually developed extrema with threshold value $t_{M m_{-} m M}=1$. When the window size is small, extrema such as $\mathrm{V}_{1}, \mathrm{P}_{1}, \mathrm{P}_{2}$, and $\mathrm{P}_{3}$ remain undetected. However, increasing the window size let those points to be detected (plots (c) and (d)). Even after increasing the window size, points that have very small extrema such as $\mathrm{P}_{4}$ will de detect as an extrema.

Before applying MMS-LH, data points (plots (a) and (b) of Figure 13) were first checked for extrema with a window size three with MMS-WBF and data in plots (c) and (d) of Figure 13 were first checked for extrema with a window size nine with MMS-WBF. Point $V_{1}$ in Figure 13(a), which seems to be a valley with high crater, yet remains as unidentified. To be qualified as an extrema with higher prominence or crater, first, the extremum must be a perfect extremum. However, with $W=3, \mathrm{~V}_{1}$ is not a perfect extremum. Therefore, the rejection is logical as well as mathematically correct. Nevertheless, in Figure 13(c), point $\mathrm{V}_{1}$ is identified as a valley, because the large window size $(W=9)$ makes $\mathrm{V}_{1}$ a nearly perfect extremum. Therefore, using $W>3$ with appropriate filter criteria the method can be used for filtering extrema with low and 

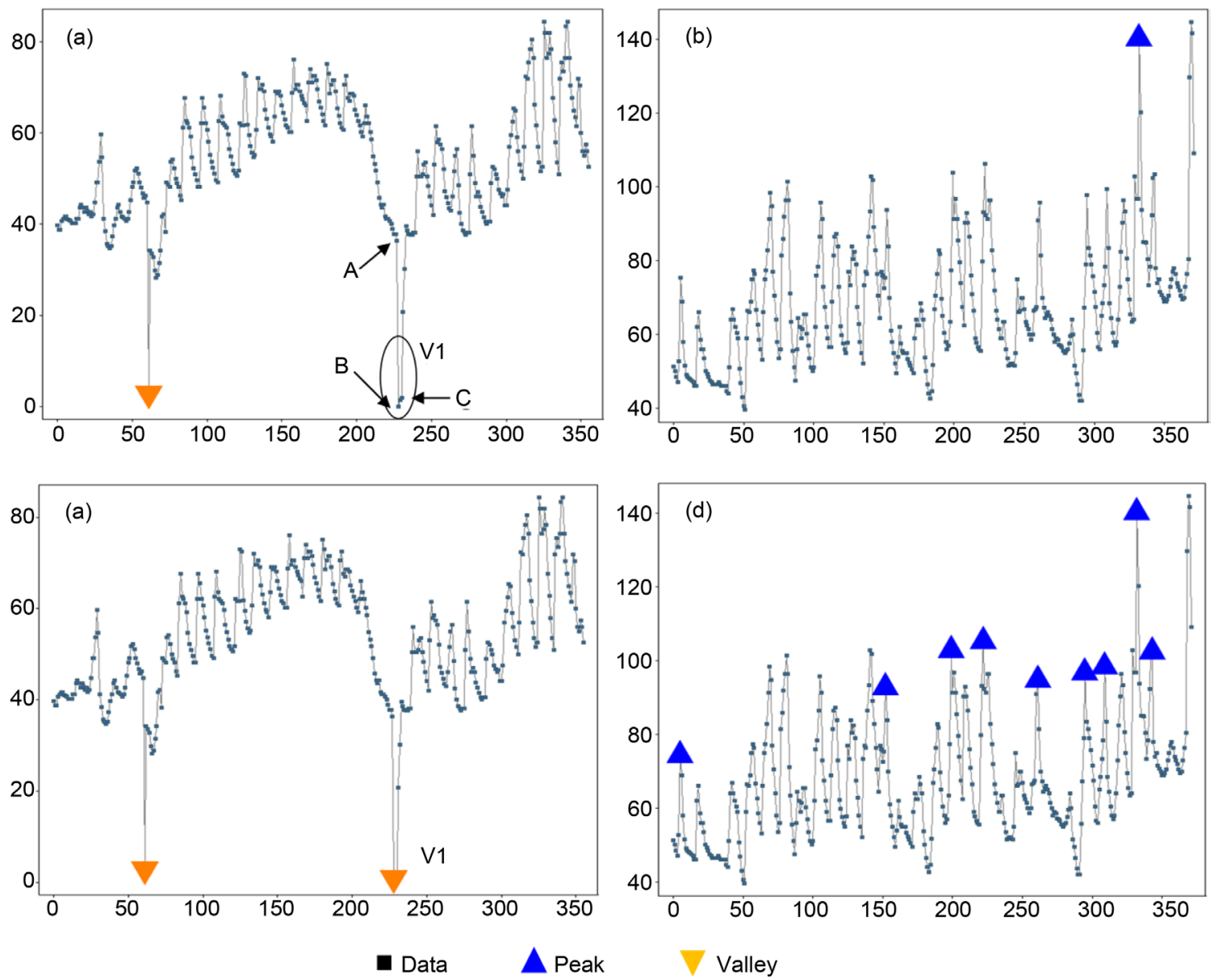

Figure 13. Filtering of low and high extrema using MMS-LH filtering technique. Data in plots (a) and (b) were first checked for extrema with a window of size three with MMS-WBF. Data in plots (c) and (d) were first checked for extrema with a window of size nine with MMS-WBF. Then $R_{L H_{-} \max }$ and $R_{L H_{-} \text {min }}$ were considered and all the plots were checked for low and high extrema with threshold value $t_{L H}=0.05$. When the window size is small, extrema such as $\mathrm{V}_{1}$ remain undetected. The reason is for such detection is that the one point (point $\mathrm{C}$ ) is located very close to the extremum (extremum is not a perfect extremum). However, increasing the window size $(W=9)$ makes $V_{1}$ a nearly perfect extremum and detected in plot (c).

high prominence or crater.

\subsection{Drawbacks of Using Large Window Size for Extrema Filtering}

In Figure 10, Figure 12, and Figure 13 plots with lager window size, $(W-1) / 2$ points from the beginning as well as from the end will not be checked, where $W$ is the window size. If there are matching extrema existing in these regions, they also remain as unidentified (Figure 12 and Figure 13). This is disadvantageous when using large window size, on the other hand if there are enough data points available, the issue is resolved. However, this is a problem for small data sets. Checking unchecked areas with a smaller window is one possibility for resolving this issue. However, results from two different window sizes will lead to violate the homogeneity of the results. The second method is to start the window before a certain number of data points $(w / 2)$. Then part of the 
window is laid on a non-data region. Using a suitable padding, this part can be filled. For example, the entire data in non-data region in the start can be padded with starting value. Also, at the end suitable padding technique can be used to fill the part of the window in the non-data region.

\subsection{Possibility of Use as a Data Segmentation Technique}

Usually, dominating peaks and the valleys can be considered as turning points of a certain property of a signal, if those dominating extrema are not outliers. Thus, dominating peaks and valleys are good points for segmenting a signal as well as identifying general trends. Figure 14 shows an attempt to accomplish such a segmenting approach using the developed method. Figure 14 contains a data set with around 4600 data points and only the MMS-WBF (dominating extrema identification technique) technique was applied as the filtering technique. For testing segmenting capabilities of the method, considerably large $W$ was used ( $W=155$ in plot (a) and $W=255$ in plot (b) of Figure 14). In both situations segmentation and general trend identification shows highly promising capabilities. Existences of more than one adjacent similar types of extrema violate the trend identification and segmentation (i.e. existence of maximum after a maximum instead of minimum). Circled areas in plot (a) of Figure 14 show two such occurrences. However, removing unnecessary adjacent peaks or valleys while keeping singular important peaks or valleys, is one solution for overcoming this problem. Thereby it is necessary to develop a methodology for removing less important extrema. Increasing the $W$ is another way of overcoming the said drawback. Plot (b) of Figure 14 shows situation of increased $W$ and detection with less adjacent same type of
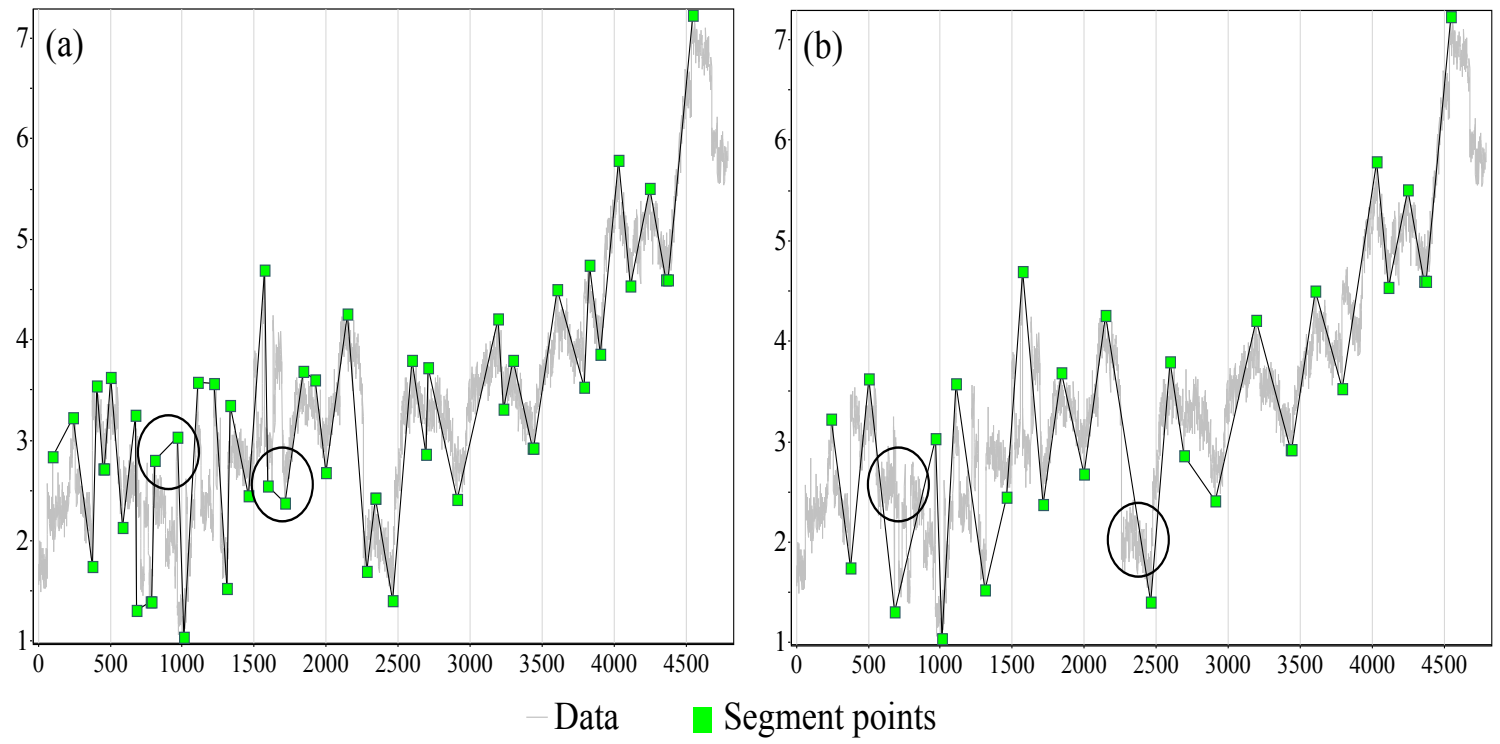

Figure 14. Usage of "MMSmax-min finder" as a segmentation technique and trend identification technique. Plot (a) and (b) use window size 155 and 255, respectively. When the window side is low ( $W=155)$ segmentation and trend identification is distracted due to occurrence of adjacent same type extrema. In plot (a) such two occurrences were circled. Increasing the window size produces better segmentation as shown in plot (b). However, this leads to ignore some trends as circled in plot (b). 
extrema than plot (a) of Figure 14. However, this technique lead to ignorance of some features in the signal as circled in plot (b) of Figure 14. Therefore, determining of proper $W$ is an essential factor for better identification of segments as well as trends. Nevertheless, the method can be used for at least fast segmentation and trend identification method.

\section{Conclusion}

The introduced extrema finding method named as "MMS Max-Min finder" and three different extrema filtering methods named as MMS-Window Based Filter (MMSWBF), MMS sharp and gradual extrema filter (MMS-SG), and MMS low high extrema filter (MMS-LH) are non-parametric. Therefore, filtering can be done without considering domain dependent parameters such as height and width of an extremum. Results prove that the detection is capable of identifying all the extrema with $0 \%$ error. When the window size is nine $(W=9)$ MMS-WBF reported $0.12 \%$ and $0.09 \%$ wrong detections. However, a combination of MMS-WBF and MMS-LH filter with window size nine ( $W=9$ ) was capable of eliminating the error. Despite of the dynamic nature of the data, the results were consistent and robust for the same detection criteria. Thus, using proper window size, it is possible to achieve robust and consistent outcome with dynamic data such as biogas data. Furthermore, MMS-WBF shows promising outcome in the direction of segmenting and trend identification of signals. Hence, MMS-WBF can be enhanced as a segmenting and trend identification technique.

\section{Acknowledgements}

This work was supported by the German Research Foundation (DFG) and the Technical University of Munich (TUM) in the framework of the Open Access Publishing Program. Also, we are grateful to the German Academic Exchange Service (Deutscher Akademischer Austauschdienst, DAAD) for providing a scholarship to KKLB Adikaram during the research period.

\section{References}

[1] Mavron, V.C. and Phillips, T.N. (2007) Maxima and Minima. In: Mavron, V.C. and Phillips, T.N., Eds., Elements of Mathematics for Economics and Finance, Springer, London, 137-158.

[2] Sande, H.V., Henrotte, F. and Hameyer, K. (2004) The Newton-Raphson Method for Solving Non-Linear and Anisotropic Time-Harmonic Problems. The International Journal for Computation and Mathematics in Electrical and Electronic Engineering, 23, 950-958. http://dx.doi.org/10.1108/03321640410553373

[3] Chioua, M., Srinivasan, B., Guay, M. and Perrier, M. (2007) Dependence of the Error in the Optimal Solution of Perturbation-Based Extremum Seeking Methods on the Excitation Frequency. The Canadian Journal of Chemical Engineering, 85, 447-453. http://dx.doi.org/10.1002/cjce.5450850407

[4] Khan, I.R. and Ohba, R. (1999) Closed-Form Expressions for the Finite Difference Approximations of First and Higher Derivatives Based on Taylor Series. Journal of Computational and Applied Mathematics, 107, 179-193. 
http://dx.doi.org/10.1016/S0377-0427(99)00088-6

[5] Gilgen, H. (2006) Univariate Time Series in Geosciences: Theory and Examples. Springer, Berlin.

[6] Zou, H.-F., Zhang, Y.-K. and Lu, P.-C. (1991) The Prediction of the Peak Width at Half Height in HPLC. Chinese Journal of Chemistry, 9, 237-244. http://dx.doi.org/10.1002/cjoc.19910090307

[7] Antoniadis, A., Bigot, J. and Lambert-Lacroix, S. (2010) Peaks Detection and Alignment for Mass Spectrometry Data. Journal de la Société Française de Statistique, 151, 17-37.

[8] Jeffries, N. (2005) Algorithms for Alignment of Mass Spectrometry Proteomic Data. Bioinformatics, 21, 3066-3073. http://dx.doi.org/10.1093/bioinformatics/bti482

[9] Sauve, A.C. and Speed, T.P. (2004) Normalization, Baseline Correction and Alignment of High-Throughput Mass Spectrometry Data. Proceedings Gensips.

[10] Mtetwa, N. and Smith, L.S. (2006) Smoothing and Thresholding in Neuronal Spike Detection. Neurocomputing, 69, 1366-1370. http://dx.doi.org/10.1016/j.neucom.2005.12.108

[11] Tzallas, A.T., Oikonomou, V.P. and Fotiadis, D. (2006) Epileptic Spike Detection Using a Kalman Filter Based Approach. IEEE Engineering in Medicine and Biology Society Conference, 1, 501-504. http://dx.doi.org/10.1109/iembs.2006.260780

[12] Gelb, A. (1974) Applied Optimal Estimation. MIT Press, Boston.

[13] Shim, B., Min, H. and Yoon, S. (2009) Nonlinear Preprocessing Method for Detecting Peaks from Gas Chromatograms. BMC Bioinformatics, 10, 378. http://dx.doi.org/10.1186/1471-2105-10-378

[14] Scholkmann, F., Boss, J. and Wolf, M. (2012) An Efficient Algorithm for Automatic Peak Detection in Noisy Periodic and Quasi-Periodic Signals. Algorithms, 5, 588-603. http://dx.doi.org/10.3390/a5040588

[15] Györfi, L., Kohler, M., Krzyzak, A. and Walk, H. (2006) A Distribution-Free Theory of Nonparametric Regression. Springer, New York.

[16] Roberts, S.J. (1997) Parametric and Non-Parametric Unsupervised Cluster Analysis. Pattern Recognition, 30, 261-272. http://dx.doi.org/10.1016/S0031-3203(96)00079-9

[17] Wasserman, L. (2006) All of Nonparametric Statistics. Springer, New York.

[18] Kothari, C.R. (2004) Research Methodology: Methods and Techniques. New Age International (P) Limited, Delhi.

[19] Li, J., Ray, S. and Lindsay, B.G. (2007) A Nonparametric Statistical Approach to Clustering via Mode Identification. Journal of Machine Learning Research, 8, 1687-1723.

[20] Adikaram, K.K.L.B., Hussein, M.A., Effenberger, M. and Becker, T. (2014) Outlier Detection Method in Linear Regression Based on Sum of Arithmetic Progression. The Scientific World Journal, 2014, Article ID: 821623. http://dx.doi.org/10.1155/2014/821623

[21] Adikaram, K.K.L.B., Hussein, M.A., Effenberger, M. and Becker, T. (2015) Universal Linear Fit Identification: A Method Independent of Data, Outliers and Noise Distribution Model and Free of Missing or Removed Data Imputation. PLoS ONE, 10, e0141486. http://dx.doi.org/10.1371/journal.pone.0141486

[22] Han, J., Kamber, M. and Pei, J. (2006) Data Mining, Southeast Asia Edition: Concepts and Techniques. Elsevier Science, Amsterdam.

[23] Shalabi, L.A., Shaaban, Z. and Kasasbeh, B. (2006) Data Mining: A Preprocessing Engine. Journal of Computer Science, 2, 735-739. http://dx.doi.org/10.3844/jcssp.2006.735.739 